\title{
EFFECT OF REGULAR PHYSICAL ACTIVITY ON NON-LIPID (NOVEL) CARDIOVASCULAR RISK FACTORS
}

\author{
${ }^{1}$ Baqiyatallah (a.s) University of Medical Sciences, Tehran, Iran \\ Sport Physiology Research Center and Department of Nutrition-Health School \\ ${ }^{2}$ Baqiyatallah (a.s) University of Medical Sciences, Tehran, Iran \\ Department of Nutrition-Health School \\ ${ }^{3}$ Shahid Beheshti University of Medical Sciences, Tehran, Iran \\ Obesity Research Center, Research Institute for Endocrine Sciences
}

MOHAMMAD REZA NAGHII ${ }^{1}$, MRARYAM ALEM AREF ${ }^{2}$, MEHDI ALMADADI ${ }^{2}$, and MEHDI HEDAYATI ${ }^{3}$

\begin{abstract}
Objectives: Cardiovascular — coronary heart disease, causing heart attack and heart failure is a leading cause of disability and death worldwide. The most important risk factors for CVD are well-established and physical activity has long been considered the cornerstone of interventions and has shown extremely important in reducing the burden of this disease. The aim of this study was to examine the association between activity and inactivity and non-lipid CVD risk factors in healthy military recruits. Materials and Methods: Selected hormones and variables such as testosterone, free testosterone, Vitamin D, homocysteine, folic acid, with the inflammatory cytokines such as hsCRP, TNF- $\alpha$ and IL- 6 were assessed in two groups (active $=50$, inactive $=50$ ), randomly matched by a questionnaire. Relationships between some measured variables and CVD risk factors were determined using simple correlation procedures. Results: Physically active subjects had significantly lower results of homocysteine $(14.3 \pm 1.9$ vs. $16.5 \pm 3.7, \mathrm{p}<0.000)$ and interleukin- $6(2.9 . \pm 5.3$ vs. $6 \pm 8.2, \mathrm{p}<0.025)$ and tended to have a nearly significantly higher free testosterone level $(13.1 \pm 7.1$ vs. $11 \pm 5.3, p=0.08)$. Significant correlations were found between BMI, WC, tHcy, and the corresponding risk factors; and also between hsCRP and IL-6, as well as TT and FT. Conclusions: Reducing the risk factors of developing CVD events such as homocysteine and interleukin-6, together with increasing free testosterone and significantly preventing its progression, clearly serve to underscore the beneficial properties of physical activity and to promote its effectiveness as a support means for healthier lifestyles in the community and, particularly, among military personnel.
\end{abstract}

Key words:

Physical activity, Non-lipid cardiovascular risk factors, Cytokines, Military personnel

\section{INTRODUCTION}

Cardiovascular disease (CVD), particularly coronary heart disease (CHD), remains a major cause of mortality, morbidity, and disability all over the world [1]. The efficacy and cost-effectiveness of various procedures, the preventive measures and medical therapies devoted to lowering individual risk factor levels and to reducing clinical CVD incidence rate are constantly improving and people live for much longer than ever before. Although progress has been made in the management of CHD for many patients, the risk for CHD remains elevated for most of them. Cardiovascular disease associated with

Received: March 25, 2011. Accepted: September 19, 2011.

Address reprint request to M.R. Naghii, No. 34, Bankmelli Alley, South Madjidieh St., Tehran, Iran, P/C 16336-54696 (e-mail: naghiimr@yahoo.com). 
dyslipidemia is a significant cause of morbidity and mortality. Low HDL-C is one of the well-established, independent and predictive risk factors for CVD. The raised level of HDL-C has been observed to be influenced by lifestyle modifications and higher activity, which should favorably result in lower CHD risk [2,3].

Although medications successfully lower LDL-C and decrease CHD risk, therapeutic lifestyle changes remain the initial therapy for most adult patients [4]. Risk reduction can be achieved through weight loss (HDL-C increases $1 \mathrm{mg} / \mathrm{dl}$ per weight loss of $3 \mathrm{~kg}$ ), diet rich in monounsaturated and polyunsaturated fatty acids (up to $5 \%$ increase), tobacco cessation (5\% to $10 \%$ increase), moderate alcohol consumption (5\% to $15 \%$ increase), and aerobic exercise (5\% to $10 \%$ increase) [5].

In view of lifestyle modifications, exercise is an important tool in lowering cholesterol, modifying lipoproteins and promoting overall health [6,7]. It can also strengthen the heart, bones, and other muscles of the body, in addition to losing body weight. The National Heart, Lung, and Blood Institute's and the National Institute of Diabetes and Digestive and Kidney Disease's guidelines recommend initial lifestyle modifications, including a reduced-calorie diet of 500-1000 calories per day, increasing physical activity, and behavioral therapy. If these changes fail to produce a result in 6 months, approved pharmacotherapy is recommended as an adjunctive means in high-risk patients [8].

Regular exercise training may be important for many patients with chronic disease, and physical activity may have differential effects depending on the specific disease, but much more research is needed to identify how regular exercise may be beneficial in maintaining the health status and in chronic disease cases. Exercise training, the major component of cardiac rehabilitation, reduces risk factors, improves functional capacity and prognosis, and enhances psychological well-being as well as the quality of life in patients suffering from CAD [9]. Recently, the American Heart Association recommended that in addition to aerobic exercise, resistance training be implemented in cardiac rehabilitation programs twice a week [10].

Given the importance of physical activity and its effect on the prevention of chronic disease, the aim of the present study was to examine the impact of physical activity on the selected non-lipid CVD risk factors in a military training installation among active and inactive male army recruits. This knowledge is critical to promote physical activity as a routine part of life and clinical practice, with particular emphasis in military personnel.

\section{MATERIALS AND METHODS}

The research design was a case-control study of active and inactive (sedentary) male army recruits who were asked to complete evaluation procedures, which included demographic and anthropometric data and an exercise questionnaire. A history of known CVD, diabetes, hypertension, and smoking habits was based on participants' self-reports on their medical history. A total of 100 male volunteers (50 cases of active and 50 controls comprising inactive personnel) within the age range of 20-40-year-olds from a military training installation participated in the study and all subjects signed a written informed consent form. The study was approved by the Ethics Committee on $\mathrm{Hu}$ man Research at the University of Medical Sciences.

Being physically active was defined as participating in 40 or more minutes of a supervised systematic exercise program of regular vigorous activity and strength training 3 days a week, plus climbing high altitudes on the third day every alternative week; at least throughout the last three months or more prior to the enrollment to the study. The type of the performed activity translates to aerobic exercises consisting of running, jogging, stretching exercises and rhythmical activity of body workouts at about 60 to $70 \%$ of maximum heart rate. In addition, half of the active subjects participated in an additional program including extra running for 3200 meters and 30 minutes of martial arts 
for 45 days within their exercise program. Inactive subjects were randomly recruited from the personnel engaged mostly in administrative works, static technical works and driving. They did not participate in any regular activity or exercise. All subjects would take their usual diet (breakfast at the worksite, lunch and dinner at home) and were abstinent from medications or supplements prior to the study. Twenty-four hours before the measurement, they refrained from physical activity. In this study, we applied the NCEP guideline to obtain an accurate picture of the current coronary heart risk factors.

Weight and height were assessed in duplicate, using a digital scale and a standard, wall-mounted stadiometer. Body mass index (BMI) was calculated as weight in kilograms divided by height in meters squared. The waist circumference was measured at the midpoint between the highest point of the iliac crest and the lowest point of the costal margin in the maxillary line, using a nonmetallic, constant-tension tape, with the subjects in light clothing. The hip or gluteal circumference was taken as the largest circumference at the posterior extension of the buttocks. The waist-to-hip ratio was obtained by dividing the waist circumference by the hip circumference. Seated blood pressure was measured; the 2 readings were averaged after a 10-minutes rest, using a mercury sphygmomanometer device.

After an overnight fasting period of 12 hours, a blood sample was drawn from an antecubital vein for measurements of total Cholesterol (TC), triglycerides (TG), high-density lipoprotein cholesterol (HDL-C), and lowdensity lipoprotein cholesterol (LDL-C). They were assayed by enzymatic colorimetric procedures with the use of commercial reagents (Parsazmun, Iran). An accredited clinical laboratory (Pasteur Medical Laboratory, Shabestar, Iran) using COBAS MIRA PLUS Auto Analyzer Devise - Switzerland completed the above analytical measurements. These measurements were used for the correlation analysis.
In addition, the selected non-lipid (novel) cardiovascular risk factors such as total testosterone (TT), free testosterone (FT), Vitamin D, homocysteine, folic acid, and the inflammatory cytokines such as high sensitive CRP (hsCRP), TNF- $\alpha$ and interleukin-6 (IL-6) were assessed in two groups (active $=50$, inactive $=50$ ).

TT, FT, and hsCRP were analyzed by ELISA, Diagnostics Biochem, Canada Inc., Ontario, Canada. The intra-assay coefficients of variation (CVs) \% and assay sensitivity were 5.1 and $0.022 \mathrm{ng} / \mathrm{ml}$ for TT; 2.0 and $0.17 \mathrm{pg} / \mathrm{ml}$ for FT; and 1.4 and $10 \mathrm{ng} / \mathrm{ml}$ for hsCRP, respectively.

25-hydroxy vitamin D was analyzed by EIA, Immuno Diagnostic Systems Ltd., Boldon, Tyne Wear, UK with the intra-assay coefficients of variation (CVs) \% and assay sensitivity of 6.9 and $5 \mathrm{nmol} / \mathrm{l}$; and IL- 6 and TNF- $\alpha$ were analyzed by ELISA, Diaclone, Besancon, France, with the intra-assay coefficients of variation (CVs) \% and assay sensitivity of 4.2 and $<2 \mathrm{pg} / \mathrm{ml}$ for IL-6, and 3.3 and $<8 \mathrm{pg} / \mathrm{ml}$ for TNF- $\alpha$, respectively. Homocysteine was analyzed by enzymatic assay method, DIAZYME Lab., Poway, USA, with the intra-assay coefficients of variation (CVs) $\%$ and assay sensitivity of 2.1 and $1.5 \mathrm{Mmol} / \mathrm{l}$. Folic acid was analyzed by Electrochemiluminescence Immunoassay, Roche Diagnostics, GmbH Germany, with the intra-assay coefficients of variation (CVs) \% and assay sensitivity of 7.6 and $0.6 \mathrm{ng} / \mathrm{ml}$.

\section{Statistical Analysis}

Data is expressed as mean \pm SD and a statistical package (SPSS 17.0) was used to perform all comparisons. Baseline characteristics of the subjects were presented as percent and mean $\pm \mathrm{SD}$ and independent sample T-tests were used for comparison of the measurements mean. Statistical significance was determined using a two-tailed $t$ test and a value of ( $p \leq 0.05$ ) was considered statistically significant. Relationships between anthropometric indices, lipids and some measured CVD risk factors were determined using simple correlation procedures. 


\section{RESULTS}

The baseline characteristics of the active and inactive participants, along with the body composition measurements and lipid profile, are shown in Tables 1 and 2. At the moment of enrollment to the study, none of the participants were smoking and reported any complaints or discomforts about cardiovascular disease. Neither were they taking any medications such as antihypertensive or lipid lowering medications.

The effects of physical activity on the lipid profile are presented in Table 2. The active group had significantly lower total cholesterol, triglycerides and LDL-cholesterol levels. The level of HDL-cholesterol was significantly higher in the active group.

Compared to sedentary men, unpaired t-test analysis showed that physically active subjects had significantly lower measures of homocysteine and interleukin- 6 and tended to have nearly significantly higher free testosterone level $(\mathrm{p}=0.08)$ (Table 3$)$.

Simple correlation coefficients between some of the traditional CVD risk factors and novel risk factors were determined. As shown in Table 4, significant correlations were found between BMI, WC, tHcy, and the corresponding variables presented in the table; and also between hsCRP and IL-6 as well as TT and FT (Table 4).

\section{DISCUSSION}

Non-communicable diseases currently represent $43 \%$ of the global burden of disease and are expected to account for $60 \%$ of the disease burden and $73 \%$ of all deaths in the world by 2020 [11]. The two leading causes of cardiac death worldwide are cardiovascular - coronary heart disease (CHD) (which causes heart attack and heart failure) and cerebrovascular disease (which causes stroke). The direct and indirect costs of CVD are high: enormous healthcare costs and productivity/income losses [12]. The World Health Organization estimates global mortality associated with $\mathrm{CHD}$ to be approximately 17 million deaths annually, representing about $30 \%$ of all the deaths worldwide. It is anticipated that CVD will become the greatest cause of morbidity and mortality in the world by the year 2015 [13]. Numerous victims of the disease, who are apparently healthy, die suddenly without prior symptoms. Available screening and diagnostic methods are insufficient to identify the victims before the event occurs.

The most important risk factors for CVD are well-established. Major risk factors caused by unhealthy lifestyles, such as hypertension, dyslipidemia, obesity, diabetes mellitus, and exposure to tobacco smoke often cluster in individual patients, dramatically increasing CVD risk [14,15]. They are strongly influenced by an unhealthy diet and physical inactivity. Prevention or treatment of risk factors for CVD is effective and sustainable in the long run. The risk of CVD can be reduced quickly and substantially with successful preventive practices.

Among the treatment strategies, drug therapy to prevent or treat CVD has focused on individual risk factors [16] and lifestyle modifications and interventions seem to be at least as effective as drugs [17] and they remain the cornerstone of CVD prevention strategies, particularly for individuals with one or more "borderline" risk factors. Intensive multifactorial interventions combining lifestyle modifications and drug therapy have the potential to dramatically reduce CVD outcomes [18], and physical activity has been proven to significantly reduce cardiovascular risk.

Until very recently in history, people necessarily engaged in regular and moderate, or at least occasional and vigorous, physical activity. However, with urbanization and industrialization, general levels of physical activity have declined. To promote and maintain health, all healthy adults need moderate-intensity aerobic (endurance) physical activity for a minimum of 30 min five days a week, or vigorous-intensity aerobic physical activity for a minimum of $20 \mathrm{~min}$ three days a week [19]. Higher levels of physical 
Table 1. Demographic information reported by the participants in the questionnaire

\begin{tabular}{lcc}
\hline \multicolumn{1}{c}{ Variable } & Inactive (Control) & Active (Case) \\
\hline Age (year) & $30.0 \pm 5.35$ & $27.2 \pm 5.1$ \\
Marital status (n) & 13 & 20 \\
$\quad$ single & 37 & 30 \\
$\quad$ married & & \\
Education (\%) & 64 & 42 \\
$\quad$ high school level & 36 & 58 \\
$\quad$ university level & none & none \\
Smoking & - & - \\
History of CVD & - & - \\
History of CVD & & - \\
$\quad$ in the family & - & - \\
CVD or other medication use & - & \\
Chest pain or discomfort & &
\end{tabular}

CVD - cardiovascular diseases.

Table 2. Physical characteristics, lipid profile of the inactive (control) and active (case) participants

\begin{tabular}{lcccc}
\hline \multicolumn{1}{c}{ Variable } & Control $(\mathrm{N}=50)$ & Case $(\mathrm{N}=50)$ & p value & Difference \\
\hline Weight $(\mathrm{kg})$ & $80.0 \pm 11.0[60-110]$ & mean $\pm \mathrm{SD}[$ range $]$ & 0.000 & 10.66 \\
Height $(\mathrm{cm})$ & $173.0 \pm 6.0[161-190]$ & $174.0 \pm 8.0[50-86]$ & 0.309 & -1.00 \\
BMI $\left(\mathrm{kg} / \mathrm{m}^{2}\right)$ & $26.60 \pm 3.0[20.6-34.0]$ & $22.70 \pm 1.90[184-195]$ & 0.000 & 3.90 \\
WC $(\mathrm{cm})$ & $94.0 \pm 8.0[76-116]]$ & $80.0 \pm 9.0[50-100]$ & 0.000 & 14.00 \\
HC $(\mathrm{cm})$ & $100.0 \pm 6.0[91-114]$ & $96.0 \pm 7.0[79-113]$ & 0.001 & 4.00 \\
WHR $(\%)$ & $0.94 \pm 0.05[0.8-1.06]$ & $0.83 \pm 0.06[0.57-0.93]$ & 0.000 & 0.11 \\
SBP $(\mathrm{mmHg})$ & $126.0 \pm 9.0[102.0-147.0]$ & $120.0 \pm 9.0[97-149]$ & 0.003 & 6.00 \\
DBP $(\mathrm{mmHg})$ & $85.0 \pm 6.0[75-97.5]$ & $80.0 \pm 6.0[60-91.5]$ & 0.000 & 5.00 \\
FBS $(\mathrm{mg} / \mathrm{dl})$ & $76.0 \pm 12.0[60-123]$ & $76.0 \pm 7.0[67-102]$ & 0.934 & -0.10 \\
TC $(\mathrm{mg} / \mathrm{dl})$ & $187.0 \pm 47.0[114-319]$ & $160.0 \pm 27.0[112-230]$ & 0.001 & 27.00 \\
LDL-C $(\mathrm{mg} / \mathrm{dl})$ & $107.0 \pm 29.0[57.0-175.0]$ & $86.0 \pm 20.0[48-138.9]$ & 0.000 & 21.00 \\
HDL-C $(\mathrm{mg} / \mathrm{dl})$ & $38.0 \pm 7.0[23.9-58.3]$ & $46.0 \pm 8.0[30.2-65]$ & 0.000 & -8.00 \\
TG $(\mathrm{mg} / \mathrm{dl})$ & $172.0 \pm 98.0[62-575]$ & $97.0 \pm 37.0[50-195]$ & 0.000 & 75.00 \\
AI & $2.80 \pm 0.61[1.55-4.66]$ & $1.92 \pm 0.57[0.81-3.11]$ & 0.000 & 0.88 \\
RF & $4.94 \pm 1.13[3.08-9.31]$ & $3.54 \pm 0.82[2.17-5.28]$ & 0.000 & 1.40 \\
\hline
\end{tabular}

BMI - body mass index, WC - waist circumference, $\mathrm{HC}$ - hip circumference, WHR - waist to hip ratio, SBP - systolic blood pressure, DBP — diastolic blood pressure, FBS — fasting blood sugar, TC - total cholesterol, LDL-C - low-density lipoprotein cholesterol, HDL-C - highdensity lipoprotein cholesterol, $\mathrm{TG}$ - triglycerides, $\mathrm{AI}$ - atherogenic index, $\mathrm{RF}$ - risk factor.

Values are expressed as the mean $\pm \mathrm{SD}$ [range]. 
Table 3. Comparison of the selected non-lipid CVD risk factors of the inactive (control) and active (case) participants

\begin{tabular}{lccc}
\hline \multicolumn{1}{c}{ Variable } & $\begin{array}{c}\text { Control }(\mathrm{N}=50) \\
\text { mean } \pm \text { SD [range] }\end{array}$ & $\begin{array}{c}\text { Case }(\mathrm{N}=50) \\
\text { mean } \pm \text { SD [range }\end{array}$ & p value \\
\hline TT $(\mathrm{ng} / \mathrm{dl})$ & $5.2 \pm 2.7[1.6-14.4]$ & $5.7 \pm 3.1[2.6-18.0]$ & 0.343 \\
FT $(\mathrm{ng} / \mathrm{dl})$ & $11.0 \pm 5.3[4.8-39.8]$ & $13.1 \pm 7.1[4.8-39.8]$ & 0.085 \\
tHcy $(\mathrm{mmol} / \mathrm{l})$ & $16.5 \pm 3.7[11.2-29.9]$ & $14.3 \pm 1.9[10.6-18.6]$ & 0.000 \\
Folic acid $(\mathrm{nmol} / \mathrm{l})$ & $7.6 \pm 2.5[4.5-21.3]$ & $7.9 \pm 2.5[5.3-20.9]$ & 0.485 \\
Vitamin D $(\mathrm{ng} / \mathrm{l})$ & $35.8 \pm 20.5[18.3-110.4]$ & $37.3 \pm 29.0[13.9-118.5]$ & 0.761 \\
hsCRP $(\mathrm{mg} / \mathrm{l})$ & $1.0 \pm 1.0[0.1-5.3]$ & $0.8 \pm 1.3[0.1-6.8]$ & 0.377 \\
TNF- $\alpha(\mathrm{pg} / \mathrm{ml})$ & $11.7 \pm 19.6[2.1-141.5]$ & $11.5 \pm 11.6[3.2-79.4]$ & 0.945 \\
IL-6 $(\mathrm{pg} / \mathrm{ml})$ & $6.0 \pm 8.2[0.2-45.4]$ & $2.9 \pm 5.3[0.3-26.0]$ & 0.025 \\
\hline
\end{tabular}

TT - total testosterone, FT - free testosterone, tHcy - total homocysteine, hSCRP - high sensitive CRP, TNF- $\alpha$ - tumor necrosis factor $\alpha$, IL-6 - interleukin-6.

Table 4. Simple correlation between anthropometric indices, lipids and CVD risk factors for the control and case groups

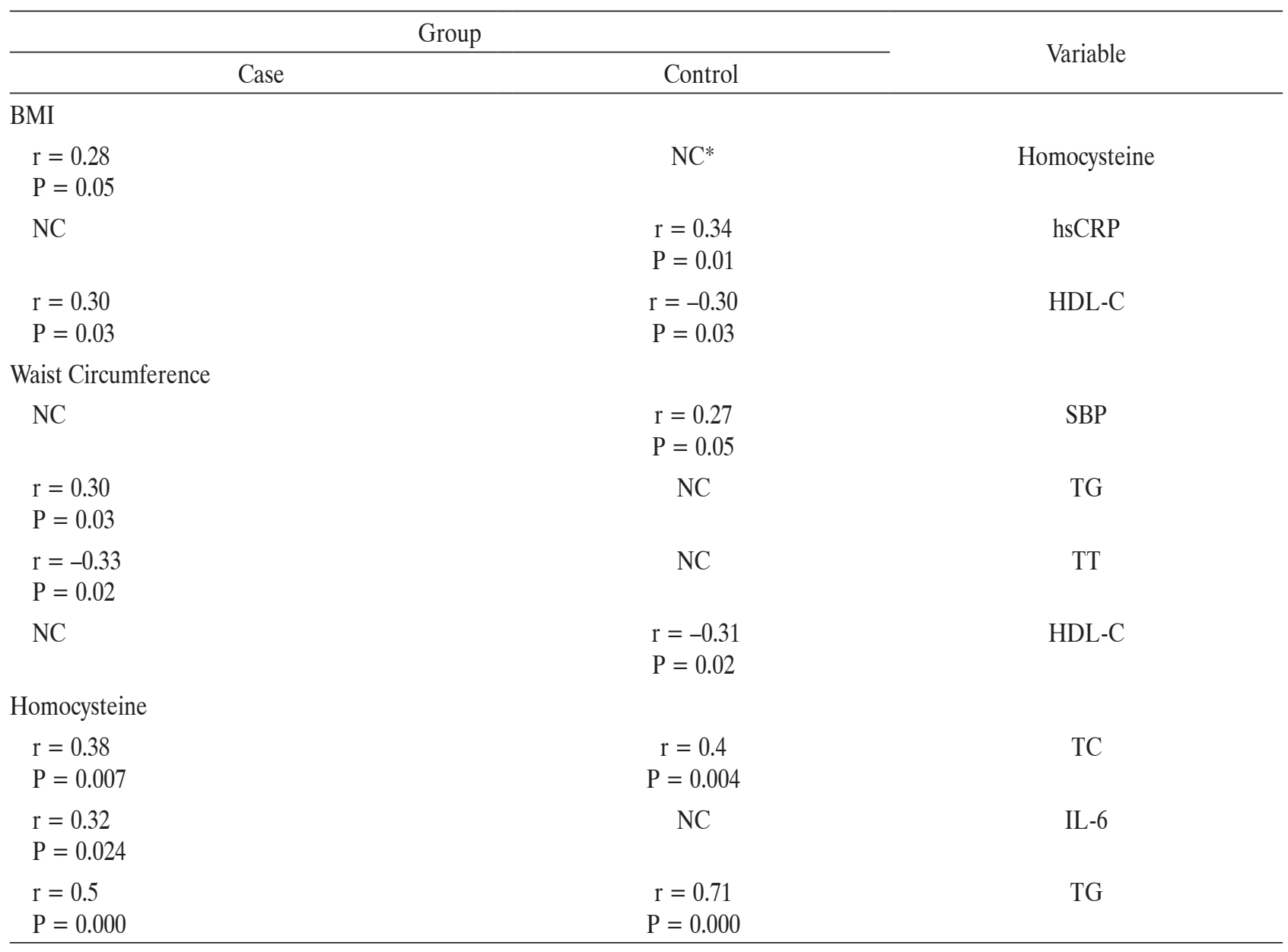


Table 4. Simple correlation between anthropometric indices, lipids and CVD risk factors for the control and case groups - cd.

\begin{tabular}{lccc} 
& Group & & Variable \\
\hline & Case & Control & HDL-C \\
\hline $\mathrm{r}=-0.33$ & $\mathrm{NC}$ & \\
$\mathrm{P}=0.02$ & & $\mathrm{IL}-6$ \\
High sensitive CRP & $\mathrm{NC}$ & \\
$\mathrm{r}=0.4$ & & $\mathrm{FT}$ \\
$\mathrm{P}=0.004$ & & \\
$\mathrm{TT}$ & $\mathrm{r}=0.76$ & \\
$\mathrm{r}=0.83$ & $\mathrm{P}=0.01$ & \\
$\mathrm{P}=0.000$ & & & \\
\hline
\end{tabular}

BMI — body mass index, hsCRP — high sensitive CRP, HDL-C — high-density lipoprotein cholesterol, SBP — systolic blood pressure, TG - triglycerides, TT - total testosterone, TC - total cholesterol, IL-6 - interleukin-6, LDL-C - low-density lipoprotein cholesterol, FT - free testosterone. $\mathrm{NC}-$ no correlation.

* Correlation is significant at the $\mathrm{p}<0.05$ level (2-tailed).

activity are associated with fewer CVD events. Although the precise mechanisms underlying this inverse association are unclear, differences in several CVD risk factors may mediate this effect [20]. Therefore, lifestyle modification, in particular physical activity long considered the cornerstone of interventions, is extremely important in reducing the burden of chronic diseases.

Determination to what extent the beneficial changes documented in the intervention studies are influenced by physical activity is a noteworthy and prominent subject to be considered.

In this study, cross-sectional analysis showed significant differences between active and inactive subjects with respect to anthropometric indices (except height, which is natural and completed due to the age), lipid profile, as well as systolic and diastolic blood pressure. Among the subjects, relations between lower levels of physical activity and higher levels of BMI, WC, and WHR, and between lipid biomarkers and significantly lower HDL were observed. These conditions have been reported to form significant associations, predominantly with the development of atherosclerosis and cardiovascular disease [21,22]. Furthermore, physically active men had lower levels of homocysteine and interleukin- 6 , and tended to have a nearly significant increase as for the free testosterone level. In the process of examining the associations of physical activity and inactivity with some of the novel risk factors, as it can be seen from the data shown in Table 4, significant positive correlations occurred between low tHcy and low BMI, TC, TG, and IL-6; and a significant negative correlation occurred as for high HDL; a significant positive correlation between high hsCRP and high BMI; and a stronger positive correlation between TT and FT in the active group. In this group, significantly lower levels of IL-6 showed a positive correlation with the lower levels of hs-CRP and tHcy, which seems to be an indication of the positive impact of physical activity on these risk factors. It is known that CRP belongs to acute-phase reactants, as its levels rise dramatically during inflammatory processes occurring in the body. This increase is due to a rise in the plasma concentration of IL-6, which is produced predominantly by macrophages [43] as well as adipocytes [44]. Moreover, as demonstrated by these results, it is clear that significant differences showed herein are the outcome of engaging in physical activity versus inactivity programs. In accordance with other reports, Guzelmeric et al. [23] and Karatela et al. [24] found higher BMI, Alipour et al. [25] higher TC, Soysal et al. [26] higher TG, Liao 
et al. [27] lower HDL, and Yun et al. [28] raised IL-6, in hyperhomocysteinemic individuals. Liao et al. [29] reported that hyperhomocysteinemia inhibits reverse cholesterol transport by reducing circulating HDL via inhibiting apo A-1 protein synthesis and enhancing HDL-C clearance. Recently, the results of the SEARCH trial has randomized over 12,000 heart attack survivors to either homocysteine-lowering therapy (with folic acid and vitamin B12) or a placebo, and followed patients for almost 7 years. It has been reported that while the increased levels of homocysteine are associated with increased cardiovascular risk, taking folic acid and B12 to reduce homocysteine levels is ineffective in reducing the risk. This indicates that homocysteine is ONLY a marker of the elevated risk, and not its cause [30]. It was found that the active group tended to have a higher folic acid levels and a significant lower total homocysteine levels.

According to $\mathrm{Su}$ et al. [31], a significant correlation was demonstrated between hsCRP and BMI. Similarly, a stronger association observed between TT and FT in the active group in this study was noted and reported by Moreno et al. [32].

The relationship between different risk factors and CVD is reported among different military recruits or forces $[33,37]$, and findings of our study have shown that higher levels of physical activity clearly and substantially lower the measures of risk factors of CVD, accounting for a substantial portion of the beneficial effect of physical activity on CVD risk reduction. Previous studies had demonstrated favorable impact of physical activity on traditional risk factors, with some individuals experiencing large changes in risk factors while engaging in exercising, and most individuals experiencing modest short-term changes in this respect [38-40]. Cardiovascular and metabolic health benefits occurred throughout 40 days vigorous physical activity and it should be encouraged even for people with risk factors and can be prescribed in a gradual increase manner [41]. It is reported that even a moderate level of physical activity (at least $600 \mathrm{kcal} / \mathrm{week}$, or the equivalent of just over $2 \mathrm{~h}$ /week of brisk walking) was identified as an underlying mechanism with the potential to lower the risk of clinically significant CVD events [20].

Data from the Behavioral Risk Factor Surveillance System indicated that from 2001 to 2005, the prevalence of regular physical activity increased by $8.6 \%$ among women and by $3.5 \%$ among men [42]. Better classic and novel measures observed in our study among active duty personnel serve as an important model for this approach.

Moreover, the deficiency in 25-hydroxyvitamin D $(25[\mathrm{OH}]$ D) is a treatable condition that has been associated with coronary artery disease and many of its risk factors $[45,46]$. Since the study was conducted in a cloudy environment with less sunshine during late fall in the north-west part of the country, no difference was observed in the Vitamin D levels among both groups, either working outdoors or indoors.

In summary, our findings indicate that major CVD (classic/traditional and novel) risk factors in our study were favorably affected by regular exercise and physical activity and were significantly different between active and inactive military personnel. These results serve to underscore the beneficial properties of physical activity engagement and suggest that behavioral interventions among sedentary individual servicemen may be required to promote the effectiveness of physical activity as a support means for healthier lifestyles. It was shown that those who were active generally had lower level of risk factors. This would reduce unhealthy risk behaviors in the community and especially among military servicemen, which would in turn decrease chronic morbidity and mortality.

Implementation of proper programs to reach these goals requires and recalls the exercise professionals reach out and work closely with healthcare providers to determine the most effective and practical way to include exercise research and/or interventions into routine care or therapeutic lifestyle changes. 


\section{REFERENCES}

1. Thelle DS. The causal role of blood lipids in the aetiology of coronary heart disease-an epidemiologist's perspective. Scand Cardiovasc J 2008;42:274-8.

2. Greenfeder S. Emerging strategies and agents to lower cardiovascular risk by increasing high density lipoprotein cholesterol levels. Curr Med Chem 2009;16:144-56.

3. Norata GD, Catapano AL. Molecular mechanisms responsible for the antiinflammatory and protective effect of $H D L$ on the endothelium. Vasc Health Risk Manag 2005;1:119-29.

4. Grundy SM, Cleeman JI, Merz CN, Brewer HB Jr, Clark LT, Hunninghake DB, et al. Implications of recent clinical trials for the National Cholesterol Education Program Adult Treatment Panel III guidelines. Circulation 2004;110:227-39.

5. Singh IM, Shishehbor MH, Ansell BJ. High-density lipoprotein as a therapeutic target: a systematic review. JAMA 2007;298: 786-98.

6. Powers E, Saultz J, Hamilton A, Lo V. Clinical inquiries. Which lifestyle interventions effectively lower LDL cholesterol? J Fam Pract 2007;56:483-5.

7. Volaklis KA, Spassis AT, Tokmakidis SP. Land versus water exercise in patients with coronary artery disease: effects on body composition, blood lipids, and physical fitness. Am Heart J 2007;154:560.e1-6.

8. National Institute of Diabetes and Digestive and Kidney Disease. Understanding Adult Obesity. National Institutes of Health (NIH) Publication No. 06-3680. November 2008. Bethesda, MD.: U.S. Department of Health and $\mathrm{Hu}-$ man Services; 2008. [2011 June 13]. Available from URL: http://win.niddk.nih.gov/publications/understanding.htm.

9. Leon AS, Franklin BA, Costa F, Balady GJ, Berra KA, Stewart KJ, et al. Cardiac rehabilitation and secondary prevention of coronary heart disease: an American Heart Association scientific statement from the Council on Clinical Cardiology. Circulation 2005;111:369-76.

10. Smith SC Jr, Allen J, Blair SN, Bonow RO, Brass LM, Fonarow $\mathrm{GC}$, et al. $A H A / A C C$ guidelines for secondary prevention for patients with coronary and other atherosclerotic vascular disease: 2006 update: endorsed by the National Heart, Lung, and Blood Institute. Circulation 2006;113:2363-72.

11. Lopez AD, Mathers CD, Ezzati M, Jamison DT, Murray CJ. Global and regional burden of disease and risk factors, 2001: systematic analysis of population health data. Lancet 2006;367:1747-57.

12. Murray CJ, Lauer JA, Hutubessy RC, Niessen L, Tomijima N, Rodgers A, et al. Effectiveness and costs of interventions to lower systolic blood pressure and cholesterol: a global and regional analysis on reduction of cardiovascular-disease risk. Lancet 2003;361:717-25.

13. Mackay J, Mensah G. The Atlas of Heart Disease and Stroke. Geneva: World Health Organization; 2004 [2011 June 13]. Available from URL: http:/www.who.int/cardiovascular_ diseases/resources/atlas/en/index.html

14. Khot UN, Khot MB, Bajzer CT, Sapp SK, Ohman EM, Brener SJ, et al. Prevalence of conventional risk factors in patients with coronary heart disease. JAMA 2003;290:898-904.

15. Gregg EW, Cheng YJ, Cadwell BL, Imperatore G, Williams DE, Flegal KM, et al. Secular trends in cardiovascular disease risk factors according to body mass index in US adults. JAMA 2005;293:1868-74.

16. Libby P. The forgotten majority: unfinished business in cardiovascular risk reduction. J Am Coll Cardiol 2005;46:1225-8.

17. Gillies CL, Abrams KR, Lambert PC, Cooper NJ, Sutton AJ, Hsu RT, et al. Pharmacological and lifestyle interventions to prevent or delay type 2 diabetes in people with impaired glucose tolerance: systematic review and meta-analysis. BMJ 2007;334:299.

18. Dzau VJ, Antman EM, Black HR, Hayes DL, Manson JE, Plutzky J, et al. The cardiovascular disease continuum validated: clinical evidence of improved patient outcomes: part I: Pathophysiology and clinical trial evidence (risk factors through stable coronary artery disease). Circulation 2006;114:2850-70.

19. Haskell WL, Lee IM, Pate RR, Powell KE, Blair SN, Franklin BA, et al. Physical activity and public health: updated recommendation for adults from the American College of Sports 
Medicine and the American Heart Association. Med Sci Sports Exerc 2007;39:1423-34.

20. Mora S, Cook N, Buring JE, Ridker PM, Lee IM. Physical activity and reduced risk of cardiovascular events: potential mediating mechanisms. Circulation 2007;116:2110-8.

21. Grundy SM. Obesity, metabolic syndrome, and coronary atherosclerosis. Circulation 2002;105:2696-8.

22. Carmena R, Duriez P, Fruchart JC. Atherogenic lipoprotein particles in atherosclerosis. Circulation 2004;109:1112-7.

23. Guzelmeric K, Alkan N, Pirimoglu M, Unal O, Turan C. Chronic inflammation and elevated homocysteine levels are associated with increased body mass index in women with polycystic ovary syndrome. Gynecol Endocrinol 2007;23:505-10.

24. Karatela RA, Sainani GS. Plasma homocysteine in obese, overweight and normal weight hypertensives and normotensives. Indian Heart J 2009;61:156-9.

25. Alipour M, Sohrabi D, Falah R, Heydarpour F, Mohammadi M. Effect of aerobic moderate exercise intensity and 15-F21-isoprostane concentrations in high cholesterol dietinduced atherosclerosis. Physiol Pharmacol 2007;11:199-207.

26. Soysal D, Savaş S, Susam I, Cevik C, Göldeli E, Sözmen E, et al. The association of plasma homocysteine, coronary risk factors and serum nitrite in patients with coronary artery disease, vascular syndrome $x$ and healthy subjects. Anadolu Kardiyol Derg 2003;3:26-34.

27. Liao D, Yang X, Wang H. Hyperhomocysteinemia and highdensity lipoprotein metabolism in cardiovascular disease. Clin Chem Lab Med 2007;45:1652-9.

28. Yun J, Kim JY, Kim OY, et al. Associations of plasma homocysteine level with brachial-ankle pulse wave velocity and inflammation profile in healthy men. Nutr Metab Cardiovasc Dis 2011;21(2):136-43.

29. Liao D, Tan H, Hui R, Li Z, Jiang X, Gaubatz J, et al. Hyperhomocysteinemia decreases circulating high-density lipoprotein by inhibiting apolipoprotein A-I Protein synthesis and enhancing HDL cholesterol clearance. Circ Res 2006;99:598-606.

30. Armitage JM, Bowman L, Clarke RJ, Wallendszus K, Bulbulia R, Rahimi K, et al. Effect of homocysteine-lowering with folic acid plus vitamin B12 vs. placebo on mortality and major morbidity in myocardial infarction survivors. JAMA 2010;303:2486-94.

31. Su SC, Pei D, Hsieh CH, Hsiao FC, Wu CZ, Hung YJ. Circulating pro-inflammatory cytokines and adiponectin in young men with type 2 diabetes. Acta Diabetol 2011;48(2):113-9.

32. Moreno SA, Shyam A, Morgentaler A. Comparison of free testosterone results by analog radioimmunoassay and calculated free testosterone in an ambulatory clinical population. J Sex Med 2010;7:1948-53.

33. Al-Qahtani DA, Imtiaz ML, Shareef MM. Obesity and cardiovascular risk factors in Saudi adult soldiers. Saudi Med J 2005;26:1260-8.

34. Al-Qahtani DA, Imtiaz ML. Prevalence of metabolic syndrome in Saudi adult soldiers. Saudi Med J 2005;26:1360-6.

35. Khazale NS, Haddad F. Prevalence and characteristics of metabolic syndrome in 111 Royal Jordanian Air Force pilots. Aviat Space Environ Med 2007;78:968-72.

36. Mikkola I, Keinänen-Kiukaanniemi S, Laakso M, Jokelainen J, Härkönen P, Meyer-Rochow VB, et al. Metabolic syndrome in connection with BMI in young Finnish male adults. Diabetes Res Clin Pract 2007;76:404-9.

37. Laclaustra-Gimeno M, González-García MP, CasasnovasLenguas JA, Luengo-Fernández E, León-Latre M, PorteroPérez P, et al. Cardiovascular risk factor progression in young males at 15-year follow-up in the General Military Academy of Zaragoza (AGEMZA) Study. Rev Esp Cardiol 2006;59: 671-8.

38. Leon AS, Sanchez OA. Response of blood lipids to exercise training alone or combined with dietary intervention. Med Sci Sports Exerc 2001;33(6 Suppl):S502-15.

39. Jakicic JM, Marcus BH, Gallagher KI, Napolitano M, Lang W. Effect of exercise duration and intensity on weight loss in overweight, sedentary women: a randomized trial. JAMA 2003;290:1323-30.

40. Thompson PD, Buchner D, Pina IL, Balady GJ, Williams MA, Marcus BH, et al. Exercise and physical activity in the prevention and treatment of atherosclerotic cardiovascular disease: 
a statement from the Council on Clinical Cardiology (Subcommittee on Exercise, Rehabilitation, and Prevention) and the Council on Nutrition, Physical Activity, and Metabolism (Subcommittee on Physical Activity). Circulation 2003;107:3109-16.

41. Norton L. Vigorous physical activity for 40 days may reduce cardiovascular risk factors. Abstract 747. American College of Sports Medicine (ACSM) 55th Annual Meeting; 2008 May 28-31, Indianapolis, USA. Medscape Medical News, 2008 [2011 June 13]. Available from: http://www.medscape. com/viewarticle/575585.

42. Centers for Disease Control and Prevention (CDC). Prevalence of regular physical activity among
adults-United States, 2001 and 2005. MMWR Morb Mortal Wkly Rep 2007;56:1209-12.

43. Pepys MB, Hirschfield GM. C-reactive protein: a critical update. J Clin Invest 2003;111:1805-12.

44. Lau DC, Dhillon B, Yan H, Szmitko PE, Verma S. Adipokines: molecular links between obesity and atheroslcerosis. Am J Physiol Heart Circ Physiol 2005;288:H2031-41.

45. Lee JH, Gadi R, Spertus JA, Tang F, O’Keefe JH. Prevalence of vitamin D deficiency in patients with acute myocardial infarction. Am J Cardiol 2011;107:1636-8.

46. Thacher TD, Clarke BL. Vitamin D insufficiency. Mayo Clin Proc 2011;86:50-60.

This work is available in Open Access model and licensed under a Creative Commons Attribution-NonCommercial 3.0 Poland License - http://creativecommons.org/ licenses/by-nc/3.0/pl/deed.en. 